\title{
A variational approach to a circular hyperelastic membrane problem
}

\author{
S. Liu, J. B. Haddow, and S. Dost, Victoria, British Columbia
}

(Received March 23, 1992)

\begin{abstract}
Summary. The variational principles of nonlinear elasticity are applied to a problem of axially symmetric deformation of a uniform circular hyperelastic membrane. The supported edge of the membrane is in a horizontal plane and its radius is equal to that of the undeformed plane reference configuration, so that an initially plane unstretched membrane is subjected to a dead load due to its weight.

It is shown how the stationary complementary energy principle can be used to obtain an accurate approximate solution for the deformation and stress distribution. It is also shown how the potential energy principle can be applied to the problem and how close bounds for an energy functional can be obtained from the two theorems. Numerical results are presented for realistic properties for a rubberlike material and for two strain energy functions, the semi-linear and the neo-Hookean.
\end{abstract}

\section{Introduction}

In this paper we consider the axially symmetric deformation of an isotropic, uniform hyperelastic membrane. Initially the membrane occupies its undeformed flat reference configuration in a horizontal plane. It is simply supported at its edge and then loaded with a dead load due to its weight. This is a genuinely nonlinear problem, which cannot be linearized even when the central deflection is very small compared with the radius.

Koiter [1] used the complementary energy principle to obtain an approximate solution for the problem with the semi-linear strain energy function. The semi-linear model is valid for small finite strains and large rotations. While Koiter's solution is for small strains and, in addition, the squares of the rotations of line elements of the surface are neglected. The procedure presented in this paper is not restricted to small strains and rotations, if a suitable strain energy function is considered, and is valid even for a large central deflections, for example for a rubberlike membrane with a radius of several meters.

Lee and Shield [2] have presented a detailed discussion of the variational principles of finite deformation elasto-statics and some applications are given in their further paper [3]. In [2] the complementary energy is expressed in terms of a statically admissible deformation gradient field in order to avoid the difficulty of inverting constitutive relations which express the nominal stress tensor as a function of the deformation gradient tensor. This difficulty does not arise with the semi-linear strain energy function and, for neo-Hookean strain energy, is avoided in the present treatment by expressing the complementary energy density, $W_{c}$, as a function of principal stretches, which are found from the principal components of Biot stress which are, in turn, found from a statically admissible nominal stress field. This nominal stress field is expressed in terms of a set of parameters and an optimization procedure is used to find the stationary value of the 
complementary energy functional with respect to the parameter space. It is shown how close bounds can be obtained for the energy function, an upper bound from the stationary potential principle and a lower bound from the stationary complementary energy principle.

\section{Formulation of the problem and governing equations}

Cylindrical polar coordinates $(R, \Theta, Z)$ denote the position of a particle of the membrane, in the undeformed reference configuration, which occupies the region

$0 \leqq R \leqq B, \quad 0 \leqq \Theta<2 \pi, \quad-H / 2 \leqq Z \leqq H / 2$

The coordinates of a particle of the middle surface are given by

$r=r(R), \quad \theta=\Theta, \quad z=z(R)$,

where

$r(B)=B, \quad z(B)=0$.

It has been noted by Haughton and Ogden [4] that, in general, the middle surface in the deformed configuration is not the same material surface as $Z=0$. However, we assume that $Z=0$ is the same material surface as the middle surface in the deformed configuration since the error which results is negligible.

The surfaces $Z= \pm H / 2$ are stress free and the usual membrane approximations are adopted, consequently the stress and deformation variables are taken as averaged across the thickness. The principal stretches, $\lambda_{1}$ and $\lambda_{2}$, of the deformed middle surface, are tangential to a meridian and a circle of latitude, respectively, and are given by

$\lambda_{1}=\frac{d s}{d r}, \quad \lambda_{2}=\frac{r}{R}$

where $s$ is the distance along a meridian of the middle surface measured from the pole. It follows from (3) that

$r^{\prime}=\lambda_{1} \cos \alpha, \quad z^{\prime}=\lambda_{1} \sin \alpha, \quad \lambda_{1}=\left(z^{\prime 2}+r^{\prime 2}\right)^{1 / 2}$,

where $\alpha$ is the angle between the tangent to a meridian of the middle surface and $R$ direction as indicated in Fig. 1, and a prime denotes differentiation with respect to $R$.

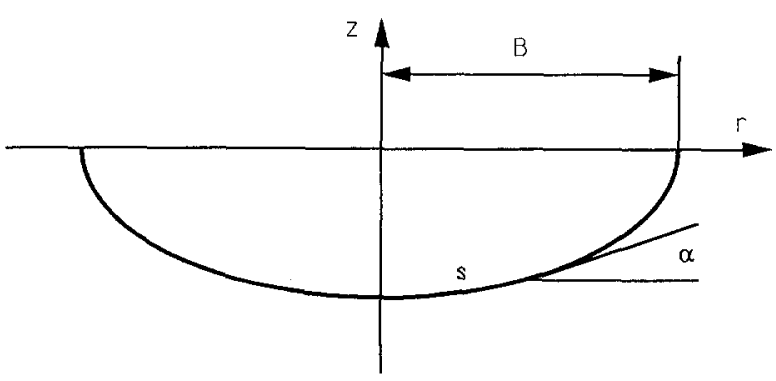

Fig. 1. Meridian of middle surface 
It follows from the membrane approximation that the nonzero components of the nominal stress tensor are $S_{R r}, S_{\Theta \theta}, S_{R z}$, and

$\frac{S_{R z}}{S_{R r}}=\tan \alpha$.

The equilibrium equations in terms of the nominal stresses are

$\frac{d S_{R r}}{d R}+\frac{S_{R r}-S_{\Theta \theta}}{R}=0$,

$\frac{d S_{R z}}{d R}+\frac{S_{R z}}{R}-\varrho g=0$,

where $\varrho$ is the density in the reference configuration. Integration of (7) gives

$S_{R z}=\varrho g R / 2$,

since the constant of integration is zero from symmetry.

We now introduce the Biot stress tensor defined by

$\mathbf{T}=\left(\mathbf{S S}^{T}\right)^{1 / 2}$,

where the superscript $T$ denotes the transpose. For the present problem the principal components of $\mathrm{T}$ are given by

$T_{1}=\left(S_{R z}^{2}+S_{R r}^{2}\right)^{1 / 2}, \quad T_{2}=S_{\Theta \theta}, \quad T_{3}=0$,

where the positive roots are taken since the principal components of membrane force per unit length, referred to the reference configuration, are given by $T_{1} H$ and $T_{2} H$ in the meridional and latitudinal directions, respectively, and these components are tensile. It follows from (5) and (9) that

$S_{R r}=T_{1} \cos \alpha, \quad S_{R z}=T_{1} \sin \alpha$.

$(10.1-2)$

\section{Constitutive relations}

The stress components $T_{1}$ and $T_{2}$ are related to $\lambda_{1}$ and $\lambda_{2}$ by relations

$T_{i}=\frac{\partial \tilde{W}}{\partial \lambda_{i}}, \quad i \in\{1,2\}$,

where $\tilde{W}\left(\lambda_{1}, \lambda_{2}\right)$ is the strain energy per unit volume of the reference configuration, for the membrane problem.

\subsection{Semi-linear model}

The strain energy function for the semi-linear model is, in terms of the principal stretches, of the same form as that for a linear elastic solid, but the principal strains $e_{i}=\lambda_{i}-1, i \in\{1,2,3\}$ are not necessarily infinitesimal. In order to compare results with those for the neo-Hookean model, we 
consider the semi-linear model with $v=0.5$, where $v$ is Poisson's ratio for infinitesimal deformation from the ground state. The resulting strain energy function, expressed as a function of the principal stretches $\lambda_{1}$ and $\lambda_{2}$, is given by

$W=\mu\left\{\left(\lambda_{1}-1\right)^{2}+\left(\lambda_{2}-1\right)^{2}+\left(\lambda_{3}-1\right)^{2}\right\}$,

with the constraint $\lambda_{1}+\lambda_{2}+\lambda_{3}=3$, where $\mu$ is the shear modulus for infinitesimal deformation from the ground state. For the present problem it is convenient to use the constraint to eliminate $\lambda_{3}$ from (12) and obtain

$\tilde{W}=2 \mu\left\{\left(\lambda_{1}-1\right)^{2}+\left(\lambda_{2}-1\right)^{2}+\left(\lambda_{1}-1\right)\left(\lambda_{2}-1\right)\right\}$.

It should be noted that the semi-linear model with $v=0.5$ does not exhibit incompressibility for finite deformation, only for infinitesimal deformation. From (11) and (13)

$T_{1}=\mu\left(4 \lambda_{1}+2 \lambda_{2}-6\right), \quad T_{2}=\mu\left(4 \lambda_{2}+2 \lambda_{1}-6\right)$,

which can easily be inverted. An explicit form

$W_{c}\left(T_{1}, T_{2}\right)=(\mu / 6)\left(T_{1}^{2}+T_{2}^{2}-T_{1} T_{2}\right)+T_{1}+T_{2}$

for the complementary energy function follows from the Legendre transformation

$W_{c}=T_{1} \lambda_{1}+T_{2} \lambda_{2}-\tilde{W}\left(\lambda_{1}, \lambda_{2}\right)$

and the inverse of (14).

\subsection{Neo-Hookean model}

The strain energy function for the neo-Hookean model is given by

$\tilde{W}=(\mu / 2)\left(\lambda_{1}^{2}+\lambda_{2}{ }^{2}+\lambda_{1}{ }^{-2} \lambda_{2}{ }^{-2}-3\right)$

and $\lambda_{3}$ is obtained from the incompressibility condition $\lambda_{1} \lambda_{2} \lambda_{3}=1$. When $T_{3}=0$, it follows from (11) and (17) that

$T_{1}=\mu\left(\lambda_{1}-\lambda_{1}^{-3} \lambda_{2}^{-2}\right), \quad T_{2}=\mu\left(\lambda_{2}-\lambda_{2}^{-3} \lambda_{1}^{-2}\right)$.

It is difficult to express the complementary energy function $W_{c}$ as an explicit function of $T_{1}$ and $T_{2}$. However, by using the Legendre transformation (16), it can be expressed in the form of

$\tilde{W}_{c}\left(\lambda_{1}, \lambda_{2}\right)=(\mu / 2)\left(\lambda_{1}^{2}+\lambda_{2}^{2}-5 \lambda_{1}^{-2} \lambda_{2}^{-2}+3\right)$

and, for given values of $T_{1}$ and $T_{2},(18)$ can be inverted numerically and a numerical value of $\tilde{W}_{c}$ obtained from (19).

\section{Variational principles}

The statements of the variational principles given in this section are for an elastic body subjected to dead loading. An extensive discussion of these principles is given by Ogden [5], and a brief outline is given in this section, for application to the membrane problem. The complementary 
energy functional for the membrane problem is given by

$E_{c}=2 \pi B^{2} H S_{R r}(B)-2 \pi H \int_{0}^{B} W_{c} R d R$

and this functional is rendered stationary by the actual stress field with respect to the set of statically admissible stress fields. If $W_{c}\left(T_{1}, T_{2}\right)$ is strictly convex the actual stress field maximizes $E_{c}$.

The potential energy functional for the membrane problem is given by

$E=2 \pi H \int_{0}^{B}(\tilde{W}+\varrho g z) R d R$

and this functional is rendered stationary by the actual deformation field with respect to the set of kinematically admissible deformation fields. If $\tilde{W}\left(\lambda_{1}, \lambda_{2}\right)$ is strictly convex the actual deformation field minimizes $E$.

For dead loading and constant body forces it is shown by Ogden [5] that

$E-E_{c}=0$

consequently if $W_{c}\left(T_{1}, T_{2}\right)$ and $\tilde{W}\left(\lambda_{1}, \lambda_{2}\right)$ are strictly convex, a chain of inequalities,

$E^{*} \geqq E=E_{c}>E_{c}{ }^{0}$

is obtained, where $E^{*}$ is the potential energy corresponding to a kinematically admissible deformation field and $E_{c}{ }^{0}$ is the complementary energy corresponding to statically admissible stress field. Upper and lower bounds, for the energy functional $E=E_{c}$, can then be obtained from (22). It is easily shown that, for the semi-linear strain energy function, $\tilde{W}$ and $W_{c}$ are strictly convex functions. For other strain energy functions it may be necessary to restrict the deformation and stress fields to subsets of the sets of kinematically admissible deformation and statically admissible stress fields. In what follows we assume that for both strain energy functions, the variational principles can be treated as extremum principles, and the numerical results obtained justify this, at least for the present problem.

In order to apply the variational principles, the following non-dimensionalization scheme is used:

$\hat{S}=S / \mu, \quad(\hat{R}, \hat{r}, \hat{z})=(R, r, z) / B$,

$\left(\hat{W}, \hat{W}_{c}\right)=\left(\tilde{W}, \tilde{W}_{c}\right) / \mu, \quad\left(\hat{E}, \hat{E}_{c}\right)=\left(E, E_{c}\right) /\left(2 \pi B^{2} H \mu\right)$.

It is convenient to introduce a non-dimensional parameter

$Q=\varrho g B /(2 \mu)$

so that the non-dimensional form of $(8)$ is

$\hat{S}_{R z}=Q \hat{R}$ 


\section{Application of complementary energy principle}

The equilibrium equations and stress boundary conditions are satisfied by stress field of the form $\hat{S}_{R r}=\sum_{i=0}^{n} a_{2 i} \hat{R}^{2 i}, \quad \hat{S}_{\Theta \theta}=\sum_{i=0}^{n} a_{2 i}(1+2 i) \hat{R}^{2 i}, \quad \hat{S}_{R z}=Q \hat{R}$,

and the corresponding Biot principal stresses are given by (9). Even terms, only, are included in polynominal expansions for $\hat{S}_{R r}$ and $\hat{S}_{\Theta \theta}$ so that the principal Biot stresses have continuous first derivatives with respect to $\hat{R}$ at $\hat{R}=0$.

The non-dimensional form of the complementary energy functional with stress field (24) is

$\hat{E}_{c}^{0}=\sum_{i=0}^{n} a_{2 i}-\int_{0}^{1} \hat{W}_{c} \hat{R} d \hat{R}$

If a set of parameters $a_{2 i}, i \in\{0,1,2 \ldots, n\}$ is chosen, then $T_{1}$ and $T_{2}$ can be evaluated at any radius $\hat{R} \in[0,1]$ and, for the semi-linear strain energy function, $\hat{W}_{c}$ can be evaluated from the non-dimensional form of (15). For the neo-Hookean strain energy function, $\hat{W}_{c}$ is evaluated by inverting (18) numerically, and substituting the resulting values of $\lambda_{1}$ and $\lambda_{2}$ in (19). Numerical integration is then used to evaluate $\hat{E}_{c}^{0}$, corresponding to the parameters $a_{2 i}, i \in\{0,1,2 \ldots, n\}$. An optimization procedure, REQPAL, developed by Chen et al. [6] is used to determine the optimized values $a_{2 i}, i \in\{0,1,2 \ldots, n\}$, which maximize $\hat{E}_{c}{ }^{0}$ with respect to the $(n+1)$ dimensional parameter space. In general the field of principal stretches obtained from the above procedure will not satisfy the boundary condition $\lambda_{2}(B)=1$, which follows from (2), but if $\lambda_{2}(B)$ differs negligibly from one, an accurate approximate solution has been obtained. The deflected form of the membrane can then be obtained from the relations

$\hat{z}=\int_{-1}^{\bar{R}} \hat{i}_{1} \hat{S}_{R z} / \hat{T}_{1} d \hat{R}$

which follows from (4.2) and (10.2) and

$\hat{r}=\int_{0}^{R} \lambda_{1} \hat{S}_{R r} / \hat{T}_{1} d \hat{R}$

which follows from (4.1) and (10.1).

\section{Application of potential energy principle}

To apply the principle of stationary potential energy, the strain energy function is expressed as a function

$W\left(r^{\prime}, z^{\prime}, r / R\right)=\tilde{W}\left\{\left(r^{\prime 2}+z^{\prime 2}\right)^{1 / 2}, r / R\right\}$,

where $r^{\prime}, z^{\prime}$ and $r / R$ by using (3.2) and (4). The non-dimensional form of the potential energy functional derived from (21) is

$\hat{E}=\int_{0}^{1} e d \hat{R}$ 
where

$e=\hat{R}\left\{\hat{W}\left(\hat{z}^{\prime}, \hat{r}^{\prime}, \hat{r} / \hat{R}\right)+2 Q \hat{z}\right\}$,

where the prime denotes derivative with respect to $\hat{R}$. Using the Euler equation, it follows

$\frac{d}{d \hat{R}}\left(\frac{\partial e}{\partial x^{\prime}}\right)-\frac{\partial e}{\partial x}=0, \quad x \in\{\hat{r}, \hat{z}\}$.

Equations (29) and (30) give two coupled nonlinear partial differential equations which appear to be untractable. We use the Euler equation with $x=\hat{z}$ which is

$\frac{d}{d \hat{R}}\left(\hat{R} \frac{\partial \hat{W}}{\partial \hat{z}^{\prime}}\right)-Q \hat{R}=0$

which, upon integration gives

$\frac{\partial \hat{W}}{\partial \hat{z}^{\prime}}-Q \hat{R}=0$

where the constant of integration is zero since $\frac{\partial \hat{W}}{\partial \hat{z}^{\prime}}$ is bounded at $\hat{R}=0$.

A kinematically admissible deformation field is constructed by approximating $\lambda_{2}$ by the polynominal

$\lambda_{2}=r / R=\hat{r} / \hat{R}=\sum_{i=0}^{m} b_{2 i} \hat{R}^{2 i}$

with the constraint $\sum_{i=0}^{m} b_{2 i}=1$.

For a given set of parameters, $b_{2 i}, i \in\{0,1,2 \ldots, m\}, \hat{r}$ and $\hat{r}^{\prime}$ are determined at $\hat{R}=k / N$, where $k=0,1,2 \ldots, N$, and $N$ is the number of intervals divided equally between 0 and $1 . \hat{z}^{\prime}$ at each $\hat{R}$ can be calculated numerically from Eq. (31), henceforth $\hat{z}$ can be integrated, and $e$ given by (29) is evaluated. Finally the potential energy functional (28) corresponding to the choice of parameters is evaluated numerically. An optimization procedure is then used to determine the values $b_{2 i}, i \in\{0,1,2 \ldots, m\}$ which minimize $\hat{E}$ with respect to the $m$-dimensional parameters space.

\section{Numerical results}

Results are obtained for a membrane of rubberlike material with properties, density $\varrho=906.5 \mathrm{~kg} / \mathrm{m}^{2}$, shear modulus $\mu=420 \mathrm{kPa}$, and with the geometry of radius $B=1 \mathrm{~m}$, so that $Q=1.05866 \times 10^{-2}$. The parameters obtained from the optimization scheme are shown in Table 1 and 2, for the two strain energy functions, and the bounds on the energy functional are shown.

The parameters $a_{2 i}, i \in\{0,1,2\}$, given in the table, lead to $\lambda_{2}(B)=1.00027$ for the semi-linear case, and 1.00008 for the neo-Hookean case, so that the boundary condition $\lambda_{2}(B)=1$ is approximately satisfied to a very high degree of accuracy, therefore the proposed application of the complementary variational principle results in a very accurate solution for both strain energy functions. 
Table 1. Results for semi-linear material

\begin{tabular}{llll}
\hline$a_{0}$ & $5.09140 \times 10^{-2}$ & $b_{0}$ & 1.0086 \\
\hline$a_{2}$ & $-9.4276 \times 10^{-3}$ & $b_{2}$ & $-8.772 \times 10^{-3}$ \\
\hline$a_{4}$ & $-9.2415 \times 10^{-5}$ & $b_{4}$ & $1.7200 \times 10^{-4}$ \\
\hline$\hat{E}_{c}^{0}$ & $-4.6792 \times 10^{-4}$ & $\hat{E}^{*}$ & $-4.6783 \times 10^{-4}$ \\
\hline$\lambda_{2}(B)$ & 1.000269 & $\lambda_{2}(B)$ & 1 \\
\hline
\end{tabular}

Table 2. Results for neo-Hookean material

\begin{tabular}{llll}
\hline$a_{0}$ & $5.0575 \times 10^{-2}$ & $b_{0}$ & 1.0088 \\
\hline$a_{2}$ & $-9.3776 \times 10^{-3}$ & $b_{0}$ & $-8.888 \times 10^{-3}$ \\
\hline$a_{4}$ & $2.2277 \times 10^{-4}$ & $b_{4}$ & $8.800 \times 10^{-5}$ \\
\hline$\hat{E}_{c}^{0}$ & $-4.6930 \times 10^{-4}$ & $\hat{E}^{*}$ & $-4.6718 \times 10^{-4}$ \\
\hline$\lambda_{2}(B)$ & 1.000083 & $\lambda_{2}(B)$ & 1 \\
\hline
\end{tabular}

It is evident that the bounds obtained for the quantity $E_{c}=E$ are very close especially for the semi-linear case.

The deflected forms obtained from the application of the complementary energy principle are shown in Fig. 2 and the distribution of stretch and principal Biot stress components are shown in Fig. 3 and 4, respectively. The deflected forms obtained from the application of the potential

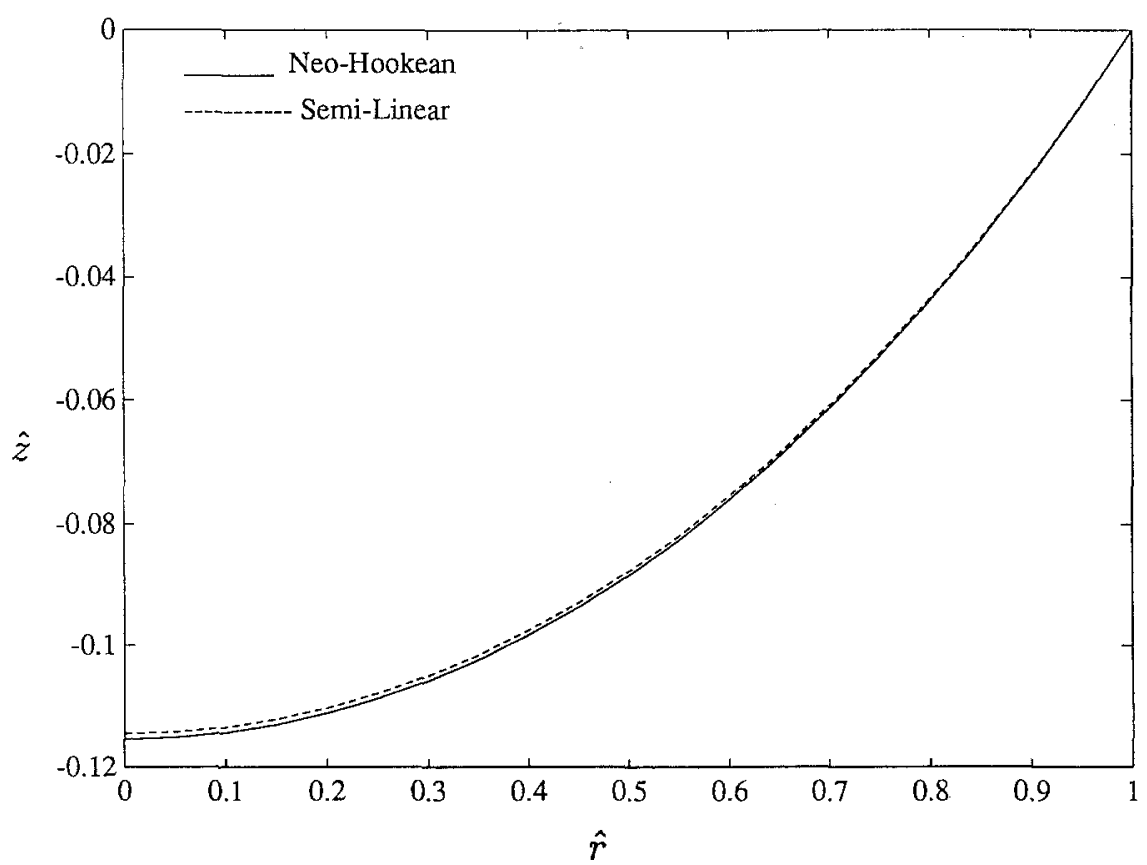

Fig. 2. Deflected form in nondimensional variables 


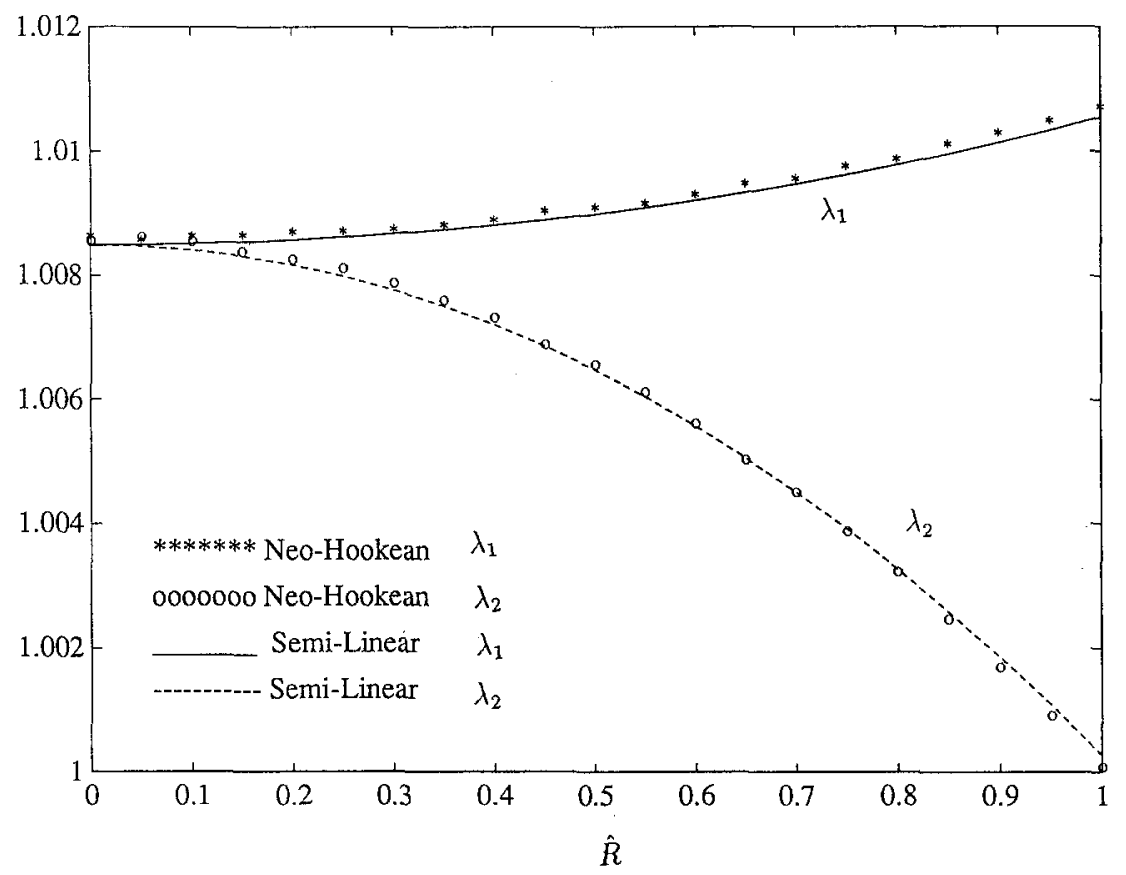

Fig. 3. Distribution of stretch

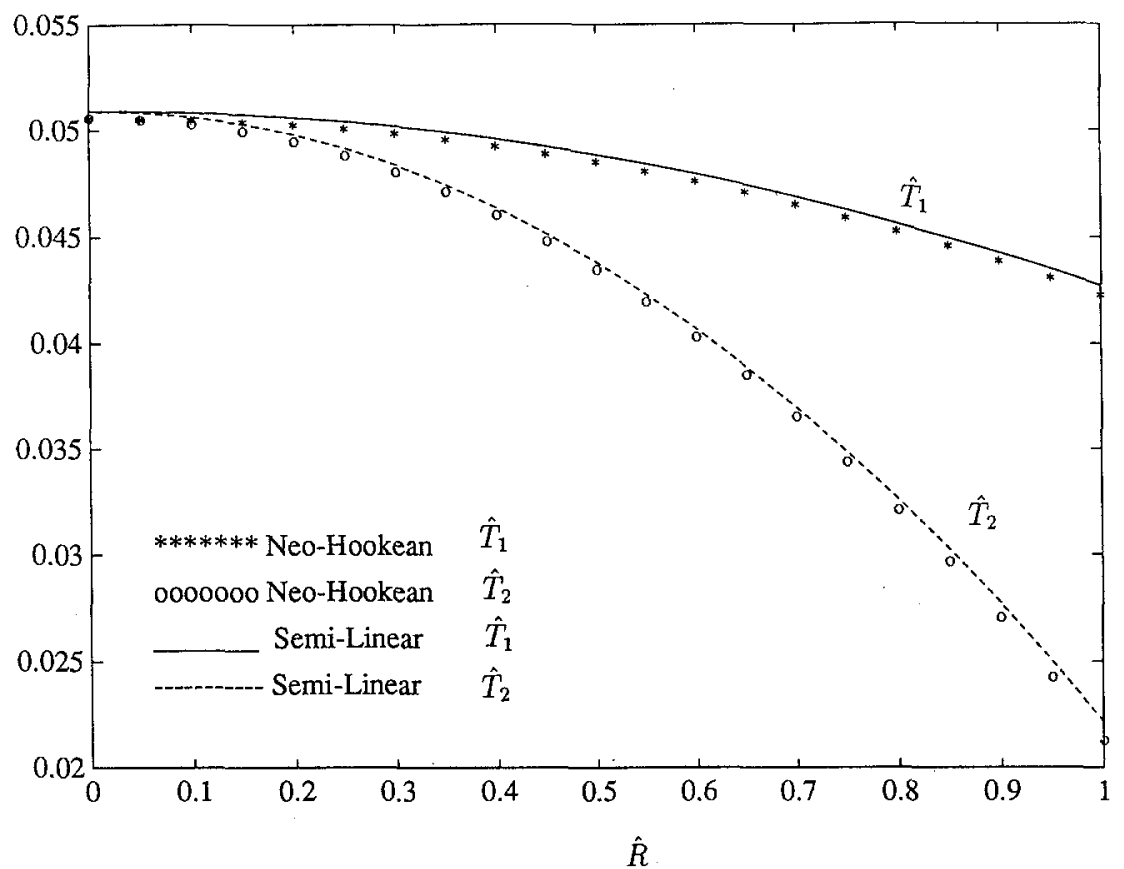

Fig. 4. Distribution of nondimensional Biot stress

energy principle differs negligibly from that shown in Fig. 2, for the semi-linear case, but for the neo-Hookean case, the central deflection is about $2 \%$ less than that indicated in Fig. 3. Possibly better agreement would be obtained if more terms in series expressed by (32) since only two independent parameters involved. 


\section{Concluding remarks}

The results shown in Fig. 2 indicate that the deflection is slightly greater for the neo-Hookean model but the difference is small for the radius considered. This is as expected since the semi-linear model requires slightly higher stresses to produce given stretches in biaxial tension.

It is surprisingly interesting to note that, for the density and shear modulus considered, which are realistic for rubberlike materials, a central deflection of approximately $11.5 \mathrm{~cm}$ is predicted for a radius of one meter.

\section{Acknowledgement}

This work is sponsored by the Natural Sciences and Engineering Research Council of Canada, through Grants No. OGP009918 and OGP0044589.

\section{References}

[1] Koiter, W. T.: On the complementary energy theorem in non-linear elasticity. In: Trends in application of pure mathematics (Fichera, G., ed.), pp. 207 -232. London: Pitman Publ. 1975.

[2] Lee, S. J., Shield, R. T.: Variational principles in finite elasto-statics. ZAMP 31 (4), 437-453 (1980).

[3] Lee, S. J., Shield, R. T.: Applications of variational principles in finite elasticity. ZAMP 31 (4), 454-472 (1980).

[4] Haughton, D. M., Ogden, R. W.: On the incremental equations in non-linear elasticity - I. Membrane theory. J. Mech. Phys. Solids 26, 93-110 (1978).

[5] Ogden, R. W.: Non-linear elastic deformations. Chichester: Ellis Horwood 1984.

[6] Chen, C., Kong, W. C., Cha, J. Z.: An equality constrained RQP algorithm based on the augmented Lagrangian penalty function. ASME J. Mech. Trans. Autom. Design 111, 368-375 (1989).

Authors' addresses: S. Liu, Department of Mechanical Engineering and Applied Mechanics, University of Michigan, Ann Arbor, MI 48109-2125, U.S.A., and J. B. Haddow and S. Dost, Department of Mechanical Engineering, University of Victoria, Victoria, British Columbia, V8W 2Y2, Canada 